\title{
A Mechanism for Vocal-Respiratory Coupling in the Mammalian Parabrachial Nucleus
}

\author{
Michael Smotherman, ${ }^{1,3}$ Kohta Kobayasi, ${ }^{1}$ Jie Ma, ${ }^{1}$ Shuyi Zhang, ${ }^{4,5}$ Walter Metzner ${ }^{1,2}$ \\ ${ }^{1}$ Department of Physiological Science and ${ }^{2}$ Brain Research Institute, University of California, Los Angeles, Los Angeles, California 90095, ${ }^{3}$ Department of \\ Biology, Texas A\&M University, College Station, Texas 77845-3258, ${ }^{4}$ Institute of Zoology, Chinese Academy of Sciences, Beijing 100080, China, and 5 \\ Guangzhou Institute of Biomedicine and Health, Chinese Academy of Sciences, Guangzhou 510663, China
}

\begin{abstract}
Mammalian vocalizations require the precise coordination of separate laryngeal and respiratory motor pathways. Precisely how and where in the brain vocal motor patterns interact with respiratory rhythm control is unknown. The parabrachial nucleus (PB) is known to mediate key respiratory reflexes and is also considered a principle component of the mammalian vocal motor pathway, making it a likely site for vocal-respiratory interactions, yet a specific role for the PB in vocalizing has yet to be demonstrated. To investigate the role of the PB in vocal-respiratory coordination, we pharmacologically manipulated synaptic activity in the PB while spontaneously vocalizing horseshoe bats were provoked to emit either short, single syllable or long, multisyllabic vocal motor patterns. Iontophoresis of the $\mathrm{GABA}_{\mathrm{A}}$ agonist muscimol (MUS) into the lateral PB extended expiratory durations surrounding all vocalizations and increased mean call durations. Alternatively, application of the $\mathrm{GABA}_{\mathrm{A}}$ antagonist bicuculline methiodide (BIC) shortened expirations and call durations. In addition, BIC eliminated the occurrence of multisyllabic vocalizations. BIC caused a mild increase in quiet breathing rates, whereas MUS tended to slow quiet breathing. The results indicate that $\mathrm{GABA}_{\mathrm{A}}$ receptor-mediated inhibition in the lateral PB modulates the time course of respiratory phase switching during vocalizing, and is needed for proper coordination of calling and breathing in mammals. We hypothesize that vocal-respiratory rhythm entrainment is achieved at least in part via mechanisms similar to other forms of locomotorrespiratory coupling, namely somatosensory feedback influences on respiratory phase-switching in the lateral $\mathrm{PB}$.
\end{abstract}

Key words: respiration; vocalization; echolocation; sensorimotor; parabrachial; speech

\section{Introduction}

Speech requires both a feedforward integration of central vocal motor commands into the respiratory cycle as well as the incorporation of somatosensory feedback onto the respiratory and vocal motor pathways (Sakamoto et al., 1997; Jürgens, 2002; Sturdy et al., 2003). Through central pathways, the vocal motor system may usurp control over the respiratory musculature for short periods of time, but over longer time spans, the temporal dynamics of speech rely on a continuous flow of pulmonary and laryngeal proprioceptive feedback to seamlessly interweave vocalizations with respiration (Kent, 1997; Nakazawa et al., 1997; Suthers et al., 2002). Although considerable progress has been achieved toward the elucidation of the respiratory-vocal integration pathway in songbirds (Sturdy et al., 2003; Kubke et al., 2004; Ashmore et al., 2005), how and where vocal motor pathways interact with the respiratory rhythm centers in the mammalian brain is less clear.

Received 0ct. 27, 2005; revised March 28, 2006; accepted March 29, 2006.

This work was supported by National Institutes of Health Grants DC005400 (W.M.) and DC00397 (M.S.), and National Science Foundation of China Grants 30025007, 30270169, and 30370264 (S.Z.). We are particularly gratefu to the Chinese Forestry Department for issuing the export permits, and to Dr. Victor Janczewski at University of California, Los Angeles, for help with obtaining the breathing measurements.

Correspondence should be addressed to Michael Smotherman, Texas A\&M University, Biology Department, 3258 TAMU, College Station, TX 77845-3258. E-mail: smotherman@tamu.edu.

D01:10.1523/JNEUROSCI.4607-05.2006

Copyright $\odot 2006$ Society for Neuroscience $\quad$ 0270-6474/06/264860-10\$15.00/0
The lateral region of the parabrachial nucleus $(\mathrm{PB})$ is endowed with a potent influence over respiratory rhythms (Feldman and Gautier, 1976; Feldman et al., 2003) and, although it is considered a central component of the mammalian vocal motor pathway (Jürgens, 2002; Smotherman et al., 2003), its specific significance with regard to vocalizing remains obscure. The $\mathrm{PB}$ is a major neuroanatomical target of descending vocal motor commands, and it projects to both laryngeal and respiratory premotor neuronal pools (Moga and Caper, 1990; Schuller and RadtkeSchuller, 1990; Farley et al., 1992; Chamberlin and Saper, 1994; Fung and St. John, 1994; Jürgens, 2002). The PB receives somatosensory feedback regarding lung status (Feldman and Gautier, 1976; Feldman, 1986; Ezure et al., 1998) and feedback regarding the mechanical status of the larynx, including subglottic pressure, vocal fold tension, and relative positions of the laryngeal cartilages (Farley et al., 1992; Sakamoto et al., 1997; Jürgens, 2002). The $\mathrm{PB}$ plays a central role in the phenomenon known as locomotor-respiratory coupling (Bramble and Carrier, 1983), in which somatosensory feedback arising from stretch-activated receptors in the arms and legs can evoke respiratory phase switching via projections from the lateral $\mathrm{PB}$ to the ventral respiratory group (Potts and Paton, 2001; Potts et al., 2005). In the case of a running animal, entraining respiratory rhythms to locomotor patterns is presumed to increased both respiratory and locomotor efficiency by assuring that competing muscle groups don't work against each other (Bramble and Carrier, 1983). Because 
vocal and respiratory motor patterns must also be rhythmically entrained, it is possible that the mechanisms underlying locomotor-respiratory coupling might also contribute to vocalrespiratory rhythm entrainment.

Here, we present the results from a series of experiments in which iontophoresis was used to manipulate synaptic activity in the lateral PB while horseshoe bats uttered complex vocal motor patterns varying specifically in number and duration of syllables but not syllable structure. The evidence indicates that GABAmediated inhibition in the $\mathrm{PB}$ is needed for the generation of extended or multisyllabic vocal sequences. We hypothesize that GABAergic inhibition in the PB serves to postpone inspiration during vocalizing until after the completion of the vocal motor sequence, possibly by regulating activity levels in the respiratory phase-switching neurons already known to exist in this region of the PB.

\section{Materials and Methods}

The data presented here were obtained from a total of 11 male and female Greater Horseshoe bats, Rhinolophus ferrumequinum, from the People's Republic of China. Six bats were used in an initial characterization of drug effects on call temporal parameters, and five additional bats were used for repeat experiments in which breathing patterns were monitored before, during, and after drug injections. Procedures were in accordance with National Institutes of Health guidelines for experiments involving vertebrate animals and were approved by the University of California, Los Angeles, Institutional Animal Care and Use Committee. The bats used in these experiments were chosen because they vocalized spontaneously under experimental conditions and reliably responded to artificial echoes. Detailed methods regarding the physiological manipulations and behavioral tests for these experiments are identical to those published previously (Smotherman et al., 2003; Smotherman and Metzner, 2005), and will only be summarized briefly. Surgery and stereotaxic approaches follow those outlined in (Schuller et al., 1986; Metzner, 1993, 1996). The bats were allowed to recover from surgery for $3 \mathrm{~d}$ before beginning the first series of experiments, during which the bats were awake and vocalizing spontaneously.

The lateral PB is a heterogeneous assemblage of neurons potentially involved in a wide array of respiratory and laryngeal control mechanisms (Farley et al., 1992; Chamberlin and Saper, 1994; Jürgens, 2002; Feldman et al., 2003; Smotherman et al., 2003; Potts et al., 2005). As described previously (Smotherman et al., 2003), injections of the $\mathrm{GABA}_{\mathrm{A}}$ antagonist bicuculline methiodide (BIC) into the lateral PB caused the dominant second harmonic of the constant-frequency portion of the echolocation call to rise by $>1.5 \mathrm{kHz}$ while the bat was calling at rest. Depending on the proximity of the injection site to the lateral portion of the $\mathrm{PB}$ (for details regarding localization of the most sensitive regions of the $\mathrm{PB}$, see Smotherman et al. 2003), these effects stabilized within $60 \mathrm{~s}$ of the onset of the injection current and typically lasted for at least 20 min after the offset of the injection current. Oppositely, injections of GABA or the $\mathrm{GABA}_{\mathrm{A}}$ agonist muscimol (MUS) caused the frequency of calls emitted at rest to be lowered by several hundred $\mathrm{Hz}$, and the effects of MUS were stable for up to $1 \mathrm{~h}$. We used changes in call frequency during and after the injections as a reliable assessment of the efficacy and location of the injection sites and as a basis for establishing the onset and duration of the drug effects.

Iontophoresis and pharmacological agents tested. Iontophoresing electrodes were triple-barrel glass micropipettes with tips broken to inner-tip diameters of $<10 \mu \mathrm{m}$. Electrodes were filled with different transmitter agonists and antagonists, respectively. This allowed us to affect the same cell cluster with both agonists and antagonists during the same penetration. Actions caused by the different drugs ejected from one multibarrel pipette into the same brain area served as a control for one another. Results presented here describe the effects of the GABAergic drugs muscimol hydrobromide (MUS, 3-hydroxy-5-aminomethylisoxazole) and BIC. The natural transmitter GABA was not used in this study because, although injections of muscimol and GABA in the PB have similar effects

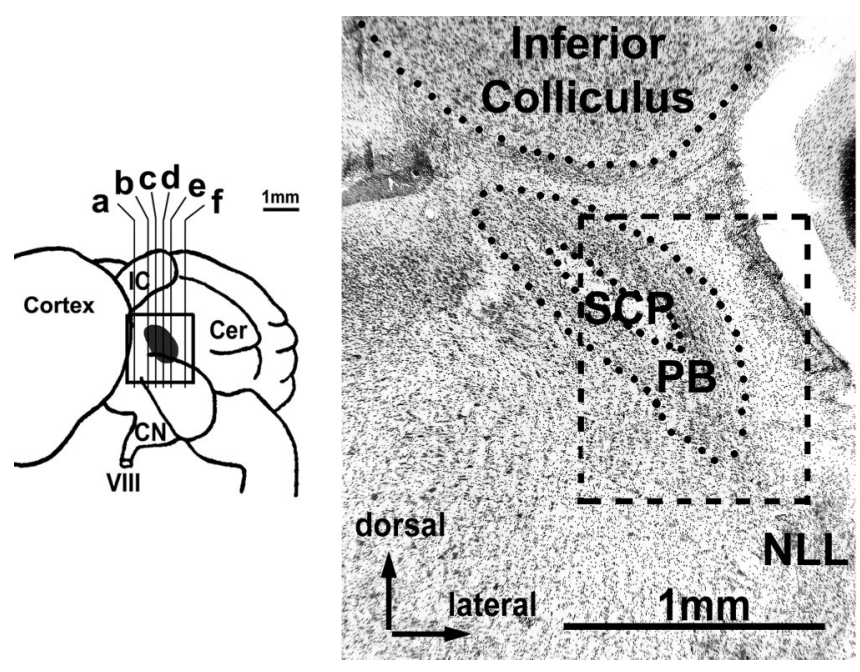

Figure 1. Reconstruction of the location of $P B$ (gray) in a side view of the horseshoe bat brain (left) and histograph of frontal section through PB (right). Letters in the left graph indicate positions of sections shown in Figure $2 A-F$. The histograph shown here corresponds to $c$. Box represents the total area covered by the reconstruction shown in Figure 2 and depicts the relative position of the PB within the area covered in Figure 2C. VIII, Auditory nerve; Cer, cerebellum; CN, cochlear nucleus; IC, inferior colliculus; NLL, nuclei of the lateral lemniscus; SCP, superior cerebellar peduncle.

on call frequency (Smotherman et al., 2003), muscimol offered longerlasting and more stable effects, which was essential for characterizing the more subtle temporal effects described here. Drugs were obtained from Sigma-RBI (St. Louis, MO). Concentrations for all agonists/antagonists were $10 \mathrm{~mm}$ in $165 \mathrm{~mm} \mathrm{NaCl}, \mathrm{pH} 3.2$, and we used $10-40 \mathrm{nA}$ positive current for injection and negative current to prevent leakage. These relatively large injection currents were necessary to produce a rapid, effective, and long-lasting result; however, injection currents alone (as determined by injecting saline at physiological $\mathrm{pH}$ ) did not elicit or suppress calling, nor did they cause permanent lesions or inhibit the return to normal after dissolution of the drug effects (Smotherman et al., 2003). Drug effects were limited to less than $\pm 200 \mu \mathrm{m}$ around the injection site (Smotherman et al., 2003).

The pipettes were placed stereotaxically in the lateral PB (Fig. 1) before drug injections and remained in place for the duration of the iontophoresis and also for the recovery period (while applying backing current) when we intended to inject the same drug again or inject a different drug from one of the two other barrels of the same triple-barrel pipette at the identical site as a control. Pipettes did not remain in the brain but were retracted at the end of each experimental session. Injection sites (Fig. 2) were verified histologically through the use of very small permanent electric lesions with an electrode at the conclusion of the experiment (Metzner, 1993) or by injecting biotinylated muscimol and visualizing the label histochemically (Smotherman et al., 2003). Comparison of the actual location of a lesion or injection site with the stereotaxically predicted position revealed an accuracy of approximately $\pm 100 \mu \mathrm{m}$. Other injection sites were reconstructed with reference to histologically verified sites using the stereotaxic coordinates of the corresponding penetrations. The nomenclature of brain structures follows those described for the rat brain (Swanson, 1992; Paxinos and Watson, 1998).

All injection sites in which the transmitter agonists and antagonists tested yielded the strongest effects were located within the lateral PB or at $<200 \mu \mathrm{m}$ distance within the midbrain tegmentum immediately adjacent to the PB (Fig. 2) as defined in Nissl-stained material. From systematically mapping the area, we estimated the area in which we could affect call frequency and temporal call parameters to cover approximately 700 $\mu \mathrm{m}$ rostrocaudally, $600 \mu \mathrm{m}$ dorsoventrally, and $300 \mu \mathrm{m}$ mediolaterally (Smotherman et al., 2003). In the following, we will refer to the lateral portions of the parabrachial nuclei and the immediately adjacent tegmentum as the lateral PB.

Acoustic playback system and behavioral test setup. All experiments were 
performed in an anechoic chamber. The electronic setup for the generation of artificial frequency-shifted echoes followed a design described previously (Schuller et al., 1974, 1975), but with modified custom-built hardware and software (Metzner et al., 2002). Calls were recorded by a quarter-inch ultrasonic microphone and amplifier (Brüel \& Kjær, Nærum, Denmark) positioned $15 \mathrm{~cm}$ in front of the bat's nostrils, electronically delayed by $4 \mathrm{~ms}$, artificially frequency-shifted (for details, see Smotherman et al. 2003), and then played back via a power amplifier (model 7500; Krohn-Hite, Avon, MA) and an ultrasonic condenser-type loudspeaker (Panasonic, Secaucus, NJ). The playback system allowed delivery of pure tone pulses of up to $122 \mathrm{~dB}$ sound pressure level (SPL), measured at the position of the bats' pinnae, over the complete range of vocalization frequencies that might typically be uttered by these bats at rest or while performing Dopplershift compensation behavior (DSC) (i.e., $74-81 \mathrm{kHz}$ ). Within the frequency range of $71-85 \mathrm{kHz}$, the playback system (including loudspeaker) had a frequency response of \pm 3 $\mathrm{dB}$ and a harmonic distortion for pure tone signals of $<60 \mathrm{~dB}$ SPL. Calibration of the playback system was performed with a quarter-inch ultrasonic microphone and power amplifier (Brüel and Kjær) using commercial signalanalysis software (Signal; Engineering Design, Berkeley, CA). The frequency and amplitude of the bats' calls were extracted from a custombuilt frequency-to-voltage and alternating/direct current converter, respectively. The accuracy for determining call frequency and amplitude was $\pm 24 \mathrm{~Hz}$ and $\pm 3 \mathrm{~dB}$, respectively. Call frequency, call amplitude, and time course and amount of induced frequency shift in the echo mimic were continuously monitored and recorded on video tape using a recording adapter (3000A; Vetter, Rebersburg, PA; sample rate $40 \mathrm{kHz} / \mathrm{chan}$ nel). Subsequent analysis was performed with the software package Datapac 2K2 (Run Technologies, Mission Viejo, CA) and commercially available statistics software (SigmaStat and SigmaPlot; Jandel, San Rafael, CA). Statistical comparisons between subsets of data were performed using the Kruskal-Wallis one-way ANOVA on ranks.

The effects of the various drugs on call frequency were determined from continuous measurements of each animal's resting frequency (RF; i.e., the frequency the bat emits and hears when not flying) and by tracking the relative change in call frequencies elicited by the playback of artificially frequency-shifted echoes. These DSC experiments included only shifts in echo frequency above the bat's RF, because the DSC behavior is highly asymmetric. Because horseshoe bats are most likely to encounter substantial increases in echo frequency during forward flight, the sensitivity of the DSC behavior is proportionally biased toward such positive Dopplershifts. Echoes were artificially frequency-shifted by slowly increasing and then decreasing the frequency of the echo playback sequentially in a sinusoidal manner. In response to this, horseshoe bats will lower and then raise their call frequency in a similarly sinusoidal manner. The rate at which playback frequency is raised and lowered is defined as the modulation frequency (Schuller et al., 1975). The maximum frequency shift presented was $3 \mathrm{kHz}$ and the modulation frequency was typically $0.1 \mathrm{~Hz}$. Intensity levels of the artificial echoes were attenuated by $10 \mathrm{~dB}$ relative to the intensity of the bat's calls.

Breathing measurements during iontophoresis and playback experiments. We initially measured breathing rates using a whole-body plethysmograph (Palecek, 1969) composed of a $100 \mathrm{ml}$ sealed Plexiglas chamber attached to an amplified ultra-low pressure sensor (model 1MBAR-D4V-REF; All Sensors, San Jose, CA) and with a constant airflow balanced to a net zero pressure difference. The whole body plethysmograph was used solely to establish baseline values for the range of breathing rates
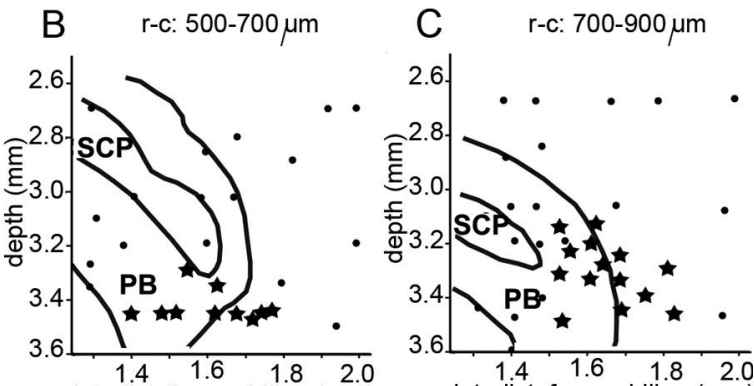

lat. dist. from midline $(\mathrm{mm})$

lat. dist. from midline $(\mathrm{mm})$
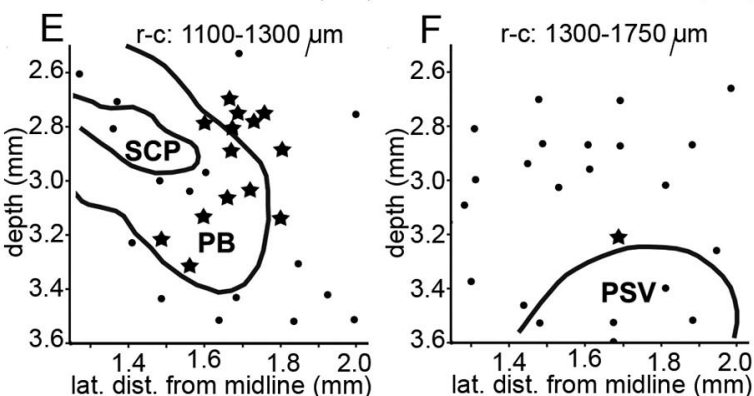

lat. dist. from midline $(\mathrm{mm})$

Figure 2. Reconstruction of sites where drug iontophoresis had marked effects on call frequencies (see also Smotherman et al., 2003, their Fig. 8) and therefore effects on temporal call parameters were analyzed as well (stars). Filled circles indicate all other a consecutive frontal sections from rostral $(\boldsymbol{A})$ to caudal $(\boldsymbol{F})$ as indicated in Figure 1. The rostrocaudal bound respectively, whereas $\boldsymbol{B}-\boldsymbol{E}$ each cover only $200 \mu \mathrm{m}$. IC, Inferior colliculus; NLL, nuclei of the lateral lemniscus; PB, parabrachial nucleus; PL, paralemniscal tegmentum; PSV, principle sensory nucleus of the trigeminal; $S C P$, superior cerebellar peduncle.

exhibited by stationary horseshoe bats, but could not be coupled to acoustic playback experiments or the in vivo electrophysiological studies.

To monitor breathing during iontophoresis of drugs into the $\mathrm{PB}$ while awake unanesthetized bats interacted with artificial acoustic playback, we relied on two noninvasive methods for monitoring gross ventilatory patterns. First, respiratory airflow was monitored using a custom-built pneumotachograph with a differential-pressure transducer (All Sensors; model 1MBAR-D-4V-REF) (Altarifi et al., 2003). The opening of a $2 \mathrm{~mm}$ inner diameter polyethylene tube was placed over the opening of one nostril and inspiration and expiration were recorded as negative and positive pressure changes within the tube, respectively. This method provided an accurate time stamp of when the direction of airflow reversed during each breathing cycle, and these data were used to calculate cycle durations as well as the time spent inspiring and expiring. However, because the opposite nostril needed to be unobstructed to allow vocalizing, as horseshoe bats emit calls through their nostrils with their mouth closed, this method could not be used to measure other quantitative ventilatory parameters such as tidal volume, minute volume, or expiratory pressure. Nevertheless, because such quantitative measurements were beyond the scope of this study, this method yielded reliable data for our purposes.

In addition to the nostril airflow measurements, we routinely used a custom-built thoracic strain gauge that was incorporated into the animal restraint system used during electrophysiological experiments. The gauge comprised an ultra low-pressure sensor attached via a $10 \mathrm{~cm}$ tube to a sealed balloon ( $2 \mathrm{~cm}$ diameter, $2 \mathrm{~mm}$ thick) filled with compressible elastic foam. The balloon was placed within a pocket of the wall of the animal restraint system (bats were restrained between a dorsal and a ventral piece of custom-fitted soft foam cut to hold the wings folded in a natural position against the side of the body wall). This foam "sandwich" was subsequently positioned against the animals flank so that expansion of the abdominal cavity during inspiration caused compression of the balloon and concomitant increase in pressure, which was registered as an increasing voltage at the output of the pressure sensor. Rapid expansion of the balloon's inner elastic foam insured that the balloon reinflated fluidly as the bat exhaled, reducing pressure in the balloon, thus, reduc- 
ing the output voltage. The restraining foam sandwich and position of the sensor were individually tailored for each bat at the beginning of each experiment to minimize imposition of any additional respiratory load. It is possible that the foam sandwich and attached strain gauge imposed a mechanical load that could have altered the respiratory rhythms away from normal; however, breathing rates measured in the foam sandwich were not significantly different from those recorded with the plethysmograph. We did not directly measure the additional respiratory load imposed by the restraining system. However, breathing rates from the beginning and ending of each experiment were compared, which established that any added load was consistent throughout the experiment. A temporal correlation and phase relationship between abdominal wall movements and nostril airflow measurements was confirmed before the initiation of each experiment.

Calculations of respiratory period, inspiration duration, and expiration duration were obtained from both the thoracic strain gauge and from measuring airflow direction at the nostrils. Measurements obtained with either method yielded results that were fully consistent with one another. Inspiration was defined as the time course of expansion of the thoracic cavity, which was tightly correlated with negative air pressure at the nostril. Expiration was defined as the period extending from the end of one inspiration to the beginning of the next. For the purpose of this study, we did not distinguish passive and active components of expiration. Bats were left undisturbed in a dark, sound-attenuated chamber for at least $20 \mathrm{~min}$ before the beginning of each recording session.

The breathing rates obtained with these methods mirrored rates obtained using the whole-body plethysmograph, and echoed results obtained with other more invasive techniques (Schuller and Rübsamen, 1981; Rübsamen and Betz, 1986; Hartley and Suthers, 1988; Suthers, 1988; Suthers et al., 1988; Schuller and Radtke-Schuller, 1990). Furthermore, the vocal temporal patterns recorded under these conditions compared favorably to earlier field and behavioral reports for naturally behaving unrestrained horseshoe bats (Schnitzler, 1968; Neuweiler et al., 1987; Tian and Schnitzler, 1997; Smotherman and Metzner, 2005), which indicates that the vocal and respiratory musculature was performing naturally during these electrophysiological studies.

\section{Results}

The results will be presented below in two parts: in the first part we present our analysis of how the iontophoresis of GABAergic drugs into the lateral $\mathrm{PB}$ altered the vocal temporal patterns of horseshoe bats responding to artificial acoustic stimuli. In the second part we present our analysis of how breathing patterns also changed during those experiments. The significance of glutamatergic synaptic activity in the lateral PB for vocal-respiratory coupling is still under investigation and will be presented in a subsequent report.

\section{Part 1}

Normal temporal dynamics of horseshoe bat vocalizations Horseshoe bat echolocation calls begin and end with transient frequency-modulated components surrounding a comparatively long constant-frequency component (Fig. 3, inset). The temporal dynamics of horseshoe bat echolocation behavior can be characterized by the presence of specific temporal pattern subsets (Smotherman and Metzner, 2005), each of which requires a different respiratory rhythm. Spontaneously calling stationary horseshoe bats (in the absence of artificial stimuli) typically emit one or fewer calls per breath (Schnitzler, 1973; Suthers, 1988). For the bats used in this study, mean call durations varied from $28 \pm 8$ to $46 \pm 12 \mathrm{~ms}$ ( $5 \mathrm{~min}$ of continuous calling per bat; mean of the means $38 \pm 9 \mathrm{~ms} ; n=11$ bats) with corresponding interpulse intervals (IPIs) ranging from 50 to $200 \mathrm{~ms}$ (mean of $135 \pm 111$ $\mathrm{ms}$ ). In horseshoe bats, the call rate is tightly coupled to the breathing rate. When horseshoe bats are presented with echo mimics (the bats own call played back through an ultrasonic

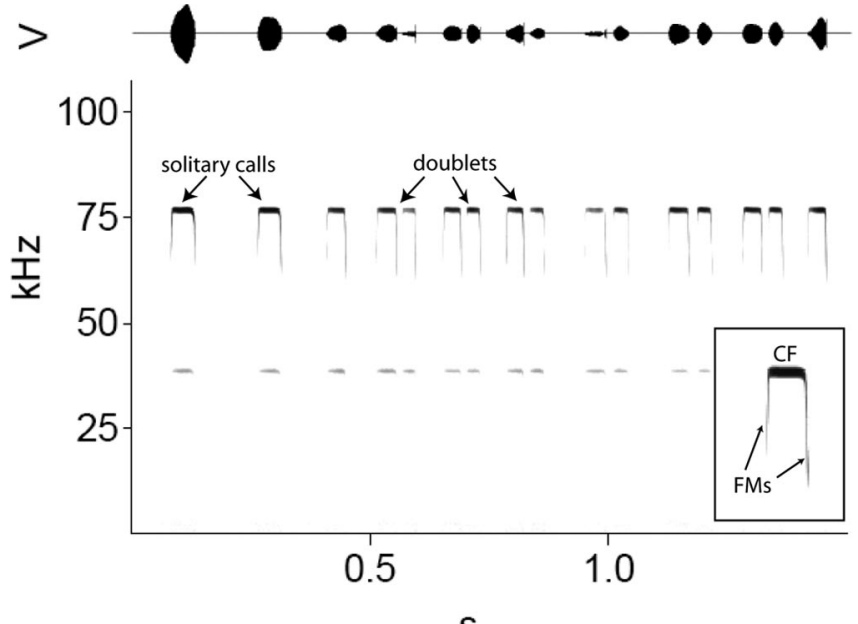

S

Figure 3. Natural variations in the temporal dynamics of call emission patterns by an echolocating horseshoe bat. Horseshoe bat calls (inset) begin and end with brief ( $<3 \mathrm{~ms}$ ) frequency-modulated (FM) components surrounding a long ( $\approx 40 \mathrm{~ms}$ ) constant-frequency (CF) component, typically $\sim 80 \mathrm{kHz}$ in Rhinolophus ferrumequinum. Calls are normally emitted once per breath, but may be emitted in pairs (doublets) during the response to specific changes in echo parameters, such as during Doppler-shift compensation behavior.

loudspeaker), which have been shifted upward in frequency to mimic the Doppler-effects that occur naturally during flight, they lower the frequency of subsequent calls in small steps until the frequency of the returning echoes returns to within a narrow bandwidth around the original call frequency (Schuller et al., 1974). During this DSC behavior (Schnitzler, 1968), horseshoe bats emit two or more calls per breath (Smotherman and Metzner, 2005). Pairs of calls are referred to as doublets (Fig. 3), and three or more calls emitted within a single breath, such as occurs naturally during feeding "buzzes," are called multiplets. The automatic doubling of call rate that occurs in response to frequency-shifted echoes accelerates the rate at which these bats can compensate for the perceived shifts in echo frequency because horseshoe bats can only make adjustments in call frequency between calls, not during calls. For this reason, call rate is a critical aspect of the compensation behavior (Schuller, 1986). During DSC behavior, mean call durations shorten by $\sim 18 \%$, reflecting the fact that the second call within a breath is usually shorter than the first call (Smotherman and Metzner, 2005). The IPIs occurring between calls within the same breath are typically $20 \mathrm{~ms}$ or less, whereas IPIs between calls separated by an inspiration are typically $>40 \mathrm{~ms}$ (Smotherman and Metzner, 2005).

\section{Effects of GABAergic drugs on vocal temporal dynamics}

We reported previously that pharmacological manipulations of GABAergic synaptic activity in the lateral PB interfered with the horseshoe bats ability to regulate call frequency during DSC (Smotherman et al., 2003). In light of evidence that horseshoe bats use a specific temporal call pattern during DSC (i.e., doublets), and that this pattern is triggered by the same acoustic cues that drive frequency compensation (Smotherman and Metzner, 2005), we were compelled to investigate whether the GABAergic drugs also affected the temporal dynamics of call emissions during DSC. Figure 4 graphs the effects of BIC and MUS injections into the lateral PB on call durations and IPIs for one representative bat (Fig. 5, bat 9), and Figure 5 compares and averages the results from six bats in cases where we were able to perform repeated tests for both BIC and MUS at precisely the same coordinates within each bat. In each case, the drug injections caused 
relative changes in call frequency and DSC performance as reported previously (Smotherman et al., 2003).

The distribution of call durations and IPIs before drug injections is illustrated as control in Figure 4, $A$ and $B$, for one bat during normal DSC performance in response to artificially frequency-shifted echoes (drug-induced changes in call frequency before and during drug injections are indicated as insets in Figure $4 B$ (Smotherman et al., 2003). During DSC, the emission of doublets caused call durations (Fig. 4A) to exhibit a bimodal distribution of short and long calls (modes at 21 and 37 ms) separated by a stereotypically short IPI, which appeared as a large mode centered around $15 \mathrm{~ms}$ in Figure 4B. Some of the interindividual variability of temporal call parameters during DSC can be gleaned from the plot comparing the results for six bats given in Figure 5, $A$ and $B$. These six individuals showed considerable variability in their frequency compensation, as well as the bimodal distribution of call durations during DSC (note that the mode for the group of longer call durations varied by $>10 \mathrm{~ms}$ between bats) and in the relative percentage of calls emitted as doublets during DSC. However, the presence of a large peak in IPIs $<40$ ms was a very consistent characteristic of all horseshoe bats performing DSC.

Injections of BIC into the $\mathrm{PB}$ elevated the bat's call frequency and abolished DSC (Fig. $4 B$, inset). Comparing periods when the bats emitted only one call per breath, BIC caused an $\sim 18 \%$ reduction in mean call durations (BIC, $33.0 \pm 8.2 \mathrm{~ms}$ vs before, $40.2 \pm 9.8 \mathrm{~ms}$; ANOVA, $p \leq 0.01$ ) and significantly decreased the mean IPI from $159 \pm 112 \mathrm{~ms}$ (before) to $131 \pm 85 \mathrm{~ms}$ (BIC). The effects of BIC on temporal call parameters during DSC are illustrated in Figure $4, C$ and $D$, for one bat, and Figure 5, $C$ and $D$, compares the effects observed in all six individuals performing DSC. Overall, the general trend was for the mean call durations to become shorter; however, BIC appears to have changed the relative distribution of call durations from broadly bimodal to a narrow unimodal distribution and eliminated the longest call durations (Figs. 2C, 3C). Pooling BIC data across bats, the mean call duration was significantly shorter than for control conditions (Fig. 5G) (BIC, $26.3 \pm 5.7 \mathrm{~ms}$ vs before, $31.3 \pm 12.8 \mathrm{~ms}$; ANOVA, $p \leq 0.01$ )

The effects of BIC on call IPIs are illustrated for a single bat in Figure $4 D$. BIC caused a conspicuous reduction in the percentage of IPIs falling below $40 \mathrm{~ms}$ (BIC, $9.7 \pm 6.4 \%$ vs before, $36.9 \pm$ $12.9 \%$; ANOVA, $p \leq 0.01$ ). BIC virtually eliminated the range of IPIs with lengths shorter than $\sim 40 \mathrm{~ms}$ (Fig. $5 C$ ), resulting in a significantly different distribution pattern of IPIs relative to the control condition (ANOVA, $p \leq 0.01$ ). For the six bats shown in Figure $5 D$, the largest percentage of IPIs typically occurred within the histogram bins ranging from 40 to $70 \mathrm{~ms}$. Simultaneously, BIC lengthened the mean IPI relative to before conditions (BIC, $155 \pm 111 \mathrm{~ms}$ vs before, $114 \pm 101 \mathrm{~ms}$; ANOVA, $p \leq 0.01)$. A
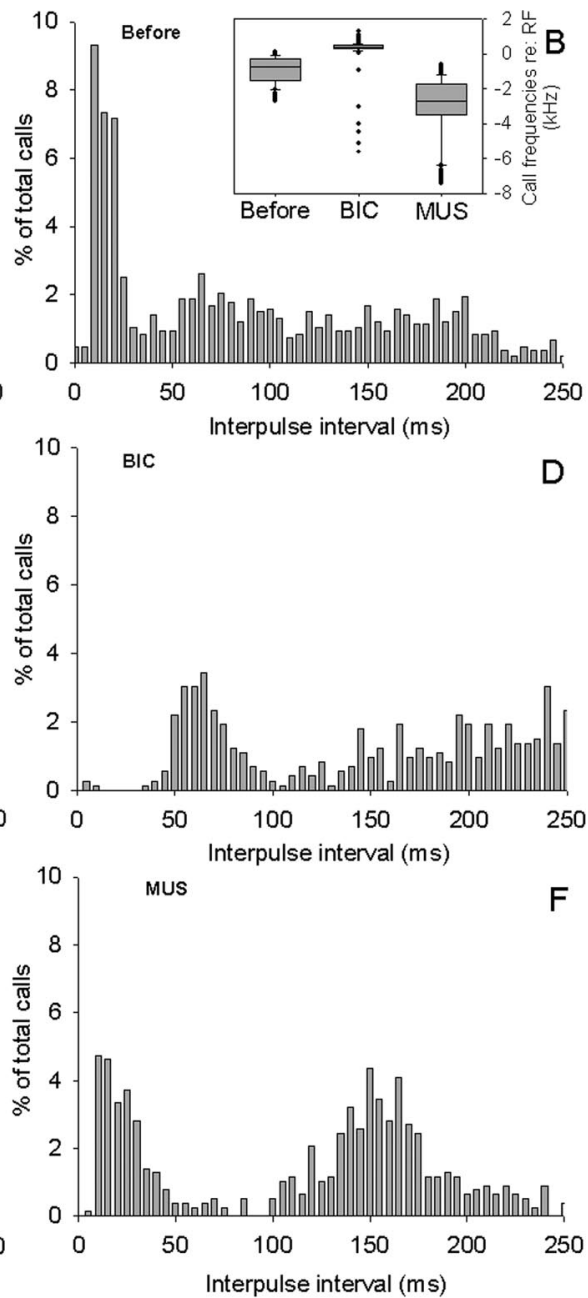

$\mathbf{F}$

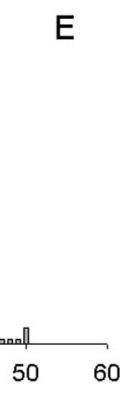

tions (right graphs) during the response to positive shifts in echo frequency (for details, see Methods and Methods) for one representative bat (bat 9 in Fig. 5). A, Distribution of call durations. $\boldsymbol{B}$, Distribution of IPIs before any pharmacological manipulations, serving as controls. The effects of BIC $(\boldsymbol{C}, \boldsymbol{D})$ and of MUS $(\boldsymbol{F}, \boldsymbol{F})$ in the same bat are shown. Inset in $\boldsymbol{B}$ shows coincident effects of the BIC and MUS injections on call frequency.

detailed look at the temporal pattern of call emissions revealed that this increase in mean IPI could be attributed to the absence of very short IPIs normally associated with the emission of doublets.

When we injected the $\mathrm{GABA}_{\mathrm{A}}$ agonist MUS into PB, we observed effects on call frequency that were opposite to those caused by BIC: during DSC, the call frequency dropped dramatically (Fig. $4 B$, inset) and the DSC behavior became generally more variable because of sporadic bouts of overcompensation. Importantly, muscimol did not eliminate DSC behavior but rather caused a downward shift in the overall range of call frequencies uttered by the bats (Smotherman et al., 2003). This result indicated to us that pharmacological manipulations of the GABAergic synaptic activity in the $\mathrm{PB}$ were altering the sensorimotor or motor control components rather than the perceptual components underlying normal DSC. The effects of muscimol on temporal call parameters were somewhat less pronounced and more variable than those caused by BIC. During periods when only single calls per breath were emitted, MUS caused no significant change in the mean call duration (MUS, $40.6 \pm 9.6 \mathrm{~ms}$ vs before, $40.2 \pm 9.8 \mathrm{~ms}$; ANOVA, $p \geq 0.15$ ) but did result in a significant increase in the mean IPIs (MUS, $172 \pm 107 \mathrm{~ms}$ vs before, $159 \pm$ $112 \mathrm{~ms}$; ANOVA, $p \leq 0.01$ ). Figure $4, E$ and $F$, illustrates the effects of MUS for one bat during DSC performance and Figure 5, 

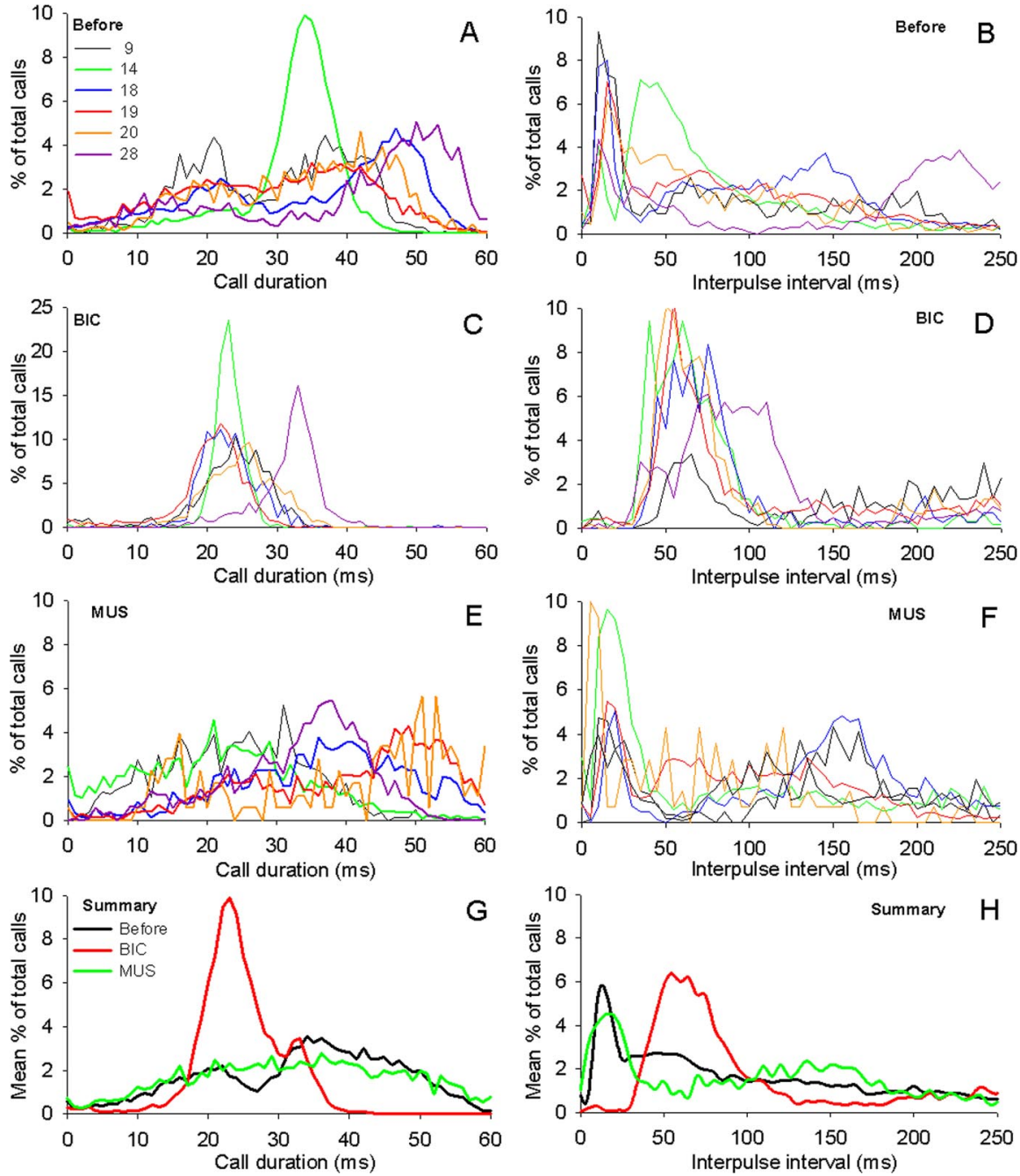

Figure 5. Summary of the effects of BIC and MUS injections on call durations (left graphs) and IPIs (right graphs) for six bats during the response to frequency-shifted echoes, graphed individually (A-F; see color key in $\boldsymbol{A}$; same convention as in Fig. 4), and averaged over all six bats $(\boldsymbol{G}, \boldsymbol{H})$. $\boldsymbol{A}, \boldsymbol{B}$, Control; $\boldsymbol{C}, \boldsymbol{D}$, effects of BIC; $\boldsymbol{E}, \boldsymbol{F}$, effects of MUS. Total number of calls included in analysis: $\boldsymbol{A}, \boldsymbol{B}, n=18,673 ; \boldsymbol{C}, \boldsymbol{D}, n=6653 ; \boldsymbol{E}, \boldsymbol{F}, n=8603 . \boldsymbol{G}, \boldsymbol{H}$, Mean effects averaged across all six bats.

$E-H$, summarizes the effects observed in six bats during DSC. Similar to BIC, MUS also caused the normally bimodal distribution of call durations to disappear (Figs. $2 E, 3 E$ ), resulting in a significantly different distribution of call durations compared with the control (one-way ANOVA, $p \leq 0.01$ ). Contrary to BIC however, on average, MUS increased the mean call duration during DSC significantly over the control value (MUS, $32.4 \pm 12.9$ ms vs before, $30.3 \pm 13.1 \mathrm{~ms}$; ANOVA, $p \leq 0.01$ ).

MUS also significantly affected the distribution of IPIs relative to the control data (ANOVA, $p \leq 0.01$ ). For the bat illustrated in Figure 4 , the mean of the IPIs after BIC injections ( $161 \pm 135 \mathrm{~ms}$ ) (Fig. $4 F$ ) was significantly different from both the IPIs recorded before the injection $(123 \pm 110 \mathrm{~ms}$; one-way ANOVA, $p \leq 0.01)$ (Fig. $4 B)$ and after BIC injections (225.4 $\pm 118.2 \mathrm{~ms}$; one-way ANOVA, $p \leq 0.001$ ) (Fig. 4D). When the effects of MUS were averaged across bats, the mean IPI was longer than controls (MUS, $134 \pm 107 \mathrm{~ms}$ vs before, $114 \pm 101$; ANOVA, $p \geq 0.05$ ). Yet, unlike the effects of BIC, MUS increased the mean IPI relative to control conditions without causing a significant reduction in the percentage of IPIs $<40 \mathrm{~ms}$ (Fig. $5 h$ ) (MUS, $33.1 \pm 16.7 \%$ vs before, $36.9 \pm 12.9 \%$; ANOVA, $p=0.18)$.

In summary (Fig. $5 G, H$ ), the distribution of temporal call parameters across all bats analyzed revealed that call durations as well as IPIs were significantly affected by iontophoretic injections of BIC and MUS into the lateral PB. BIC tended to shorten calls relative to the control condition, whereas MUS caused calls to be slightly longer on average. After injections of BIC, the appearance of short duration calls were no longer coupled to the appearance of IPIs $<40 \mathrm{~ms}$, which indicates that BIC didn't just eliminate doublet emissions, but actually caused the bats to emit an unnatural temporal call pattern. BIC and MUS both caused an increase in the mean interpulse intervals, but their effects can be traced to two separate mechanisms: mean IPIs were longer after BIC injection because of the elimination of the short "within-breath" IPIs, but the longer mean IPIs that followed MUS injections could not be attributed to the same cause; thus, it is concluded that MUS must have caused an increase in IPIs extending between doublets, not within doublets.

\section{Part 2}

Normal breathing patterns of echolocating horseshoe bats

We repeated the above experiments in five bats in which we also monitored breathing patterns before, during, and after injecting drugs into the $\mathrm{PB}$. In each case we recorded (1) quiet breathing, (2) breathing during bouts of calling in which the bats continuously emitted one call per breath, and (3) breathing during DSC, during which the bats emitted two calls per breath. In all cases the bats were awake, unanesthetized, and called spontaneously. No electrical or chemical stimuli were used to provoke calling. During quiet breathing, the average breathing rate for five bats was 5.2 breaths per second (pooling data collected from 60 s per bat, $n=1560$ total breaths; mean respiratory period was $190 \pm 60 \mathrm{~ms})$. Similar ranges $(3-8 \mathrm{~Hz})$ and mean breath rates were obtained using the whole-body plethysmograph or with either the nasal cannula or the thoracic strain gauge. Although there were large variations between individuals and across sessions, when the data from five bats were pooled, the mean time spent inspiring ( $104 \pm 43 \mathrm{~ms}$ ) was longer than the time spent expiring $(82 \pm 33 \mathrm{~ms})(p<0.001)$ (Fig. $6 A)$.

When the bats transitioned from quiet breathing to calling at a rate of one call per breath, the average breathing rate increased to $7.6 \mathrm{~Hz}$ (mean respiratory period of $131 \pm 51 \mathrm{~ms} ; 60 \mathrm{~s} / \mathrm{bat} ; n=$ 1805 total breaths; one-way ANOVA, $p<0.01$ ). The increase in breathing rate (Fig. 6B) was accounted for by a reduction in the amount of time spent inspiring between calls ( $52 \pm 26 \mathrm{~ms}$ inspirations vs quiet inspirations, $104 \pm 43 \mathrm{~ms} ; p<0.01$ ), whereas expirations remained relatively unchanged. Alternatively, when the bats performed DSC behavior (Fig. 6C), during which they emitted two rather than one call per expiration, an increase in the time spent expiring $(125 \pm 30 \mathrm{~ms}$ vs quiet expiration duration, $82 \pm 33 \mathrm{~ms}$ ) accounted for a relative decrease in the mean breath- 


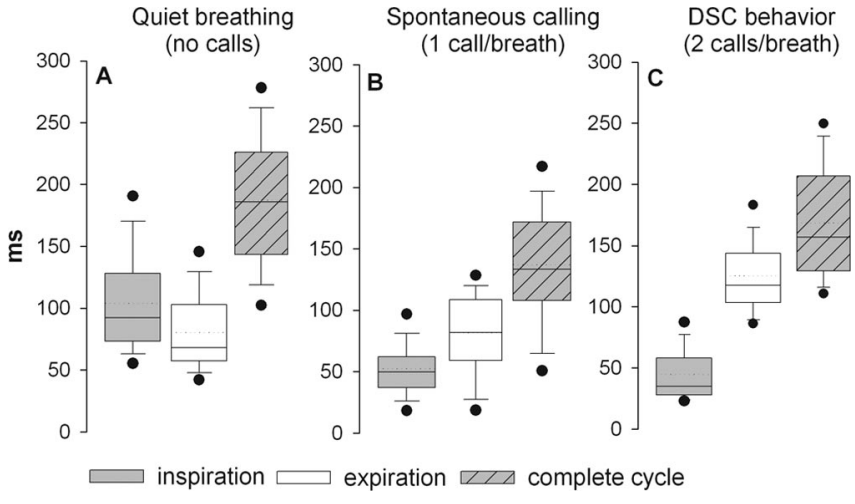

Figure 6. Ventilatory patterns measured during quiet breathing in normal horseshoe bats $(\boldsymbol{A})$ while emitting one call per breath $(\boldsymbol{B})$, and in response to simulated Doppler-shifts in the frequency of the echo mimic (i.e., during DSC behavior), which normally provoked the emission of two calls per breath $(\boldsymbol{C})$. The transition from quiet breathing to emitting one call per breath is accompanied by an abrupt shortening of the inspiratory period (compare $\boldsymbol{A}, \boldsymbol{B}$ ), whereas the transition to DSC behavior (compare $\boldsymbol{B}, \boldsymbol{C}$ ) was characterized by an increase in the expiratory period to accommodate the addition of the second call within each expiration. Data are pooled from 1 min recordings from five bats. In $\boldsymbol{A}, n=1560$ total breaths; in $B, n=1805$; in $\boldsymbol{C}, n=$ 1770. The dotted lines are means, solid lines are medians, boxes surround 1 SD, whiskers are 10th and 90th percentiles, and black dots are 5th and 95th percentiles.

ing rate (5.9 breaths per second; mean respiratory period of $160 \pm 47 \mathrm{~ms} ; n=1770$ total breaths), whereas the time spent inspiring remained unchanged relative to the breathing patterns observed while the bat emitted one call per breath. Thus, the respiratory transition from quiet breathing to emitting one call per breath was characterized by a decrease in inspiration durations, and the transition from emitting one to two calls per breath was characterized by an increase in expiration durations.

\section{Effects of GABAergic drugs on breathing patterns}

Figure 7 presents the effects of the GABAergic drugs BIC and MUS on inspiration and expiration during quiet breathing (Fig. $7 A, B)$ while emitting one call per breath (Fig. $7 C, D)$ and while emitting two calls per breath (Fig. $7 E, F$ ). Data were pooled from 1 min bins of continuous recordings for each condition from all five bats (i.e., $1 \mathrm{~min}$ of quiet breaths before injections, $1 \mathrm{~min}$ of quiet breaths after BIC injection, and 1 min of quiet breathing after MUS injection). MUS and BIC injections were performed in series at the same injection site, with at least $1 \mathrm{~h}$ separating the end of the effects of the first drug and injection of the second drug. The order of drug injection was varied in each experiment, and experiments were repeated two or more times in each bat.

BIC caused an increase in the mean quiet breathing rate from 5.3 to 6.5 breaths per second ( $p \leq 0.01$ ). This increase in breathing rate was accounted for by a significant reduction in mean inspiratory durations (Fig. 7A). Quiet inspirations after BIC injections were similar in duration to the inspirations recorded during normal calling (Fig. 7B, compare with "before" condition). BIC did not cause a significant change in quiet expiration durations. In contrast, MUS caused a significant increase in the amount of time spent expiring (Fig. $7 B)(p \leq 0.01)$, but MUS did not alter inspiration times during quiet breathing. Overall MUS caused a slight reduction of mean quiet breathing rates to $5.0 \mathrm{~Hz}$ $(p \leq 0.01)$.

During bouts of calling at one call per breath, inspiration durations were statistically indistinguishable for the before, BIC, and MUS conditions (Fig. 7C). In contrast, MUS caused a significant increase in the mean expiration duration when emitting one call per breath $(p \leq 0.01)$, whereas BIC did not have any signif-
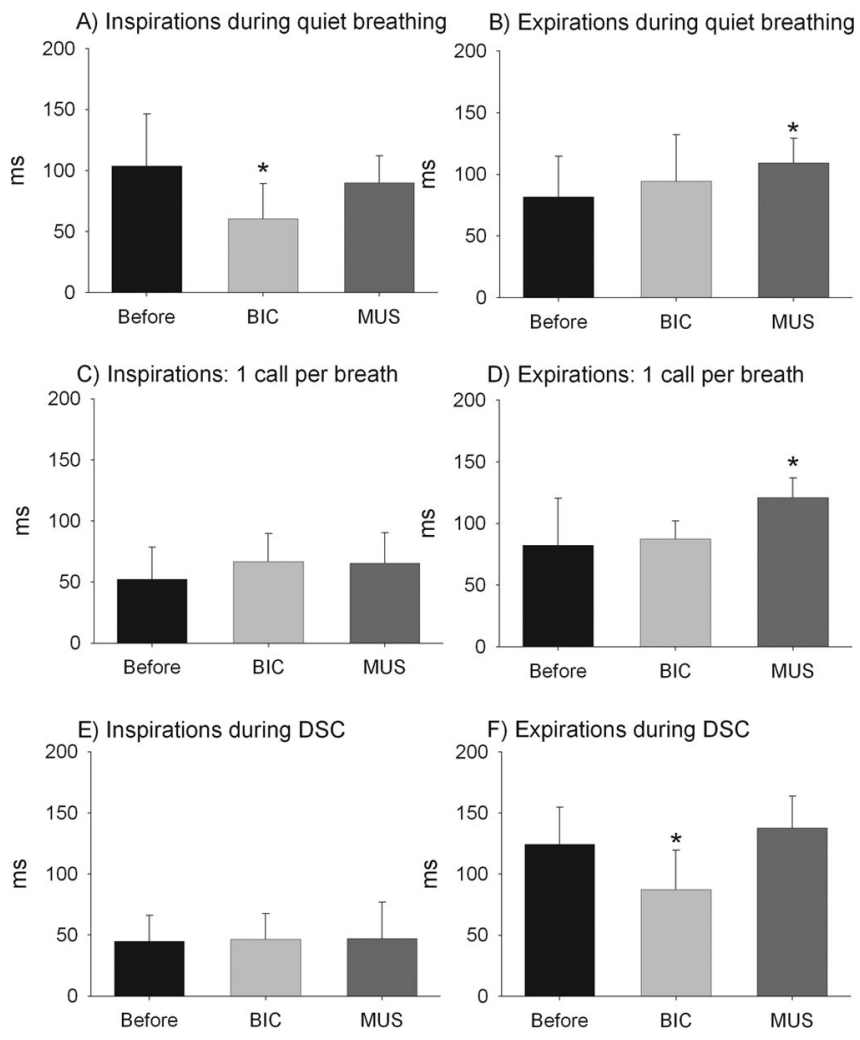

Figure 7. Comparison of ventilatory patterns for three different vocal motor patterns [quiet breathing $(\boldsymbol{A}, \boldsymbol{B})$, one call per breath $(\boldsymbol{C}, \boldsymbol{D})$, and during the response to simulated DSC $(\boldsymbol{E}, \boldsymbol{F})]$ measured before and after injections of BIC or MUS. Stars indicates a significant difference ( $p \leq$ $0.01)$ relative to the "before" condition. Data are pooled from 1 min recordings per bat per condition. The before data is the same as in Figure 6. For BIC, $n=1948(\boldsymbol{A}, \boldsymbol{B}), n=1875(\boldsymbol{C}, \boldsymbol{D})$, and $n=2160(\boldsymbol{E}, \boldsymbol{F})$ total breaths. For MUS, $n=1503(\boldsymbol{A}, \boldsymbol{B}), n=1590(\boldsymbol{C}, \boldsymbol{D})$, and $n=1644$ $(\boldsymbol{E}, \boldsymbol{F})$ total breaths. Whiskers denote 1 SD.

icant effect on expiration durations for the same call pattern (Fig. $7 D)$.

Finally, when emitting two calls per breath during DSC performance, inspiration durations were again uniformly short for all three conditions; we found no evidence that either BIC or MUS caused a statistically significant change in inspiration durations during DSC (Fig. 7E). However, BIC yielded a significant reduction in the mean expiration duration during DSC relative to the before condition (Fig. $7 F)(p \leq 0.01)$. Expiration durations for the before and MUS conditions but not for BIC increased significantly relative to the corresponding expiration durations observed when the bats were emitting one call per breath (Fig. 7, compare $F$ with $B, D)$. Thus, the results indicate that expiration durations increased normally to accommodate the second call during DSC in the before and MUS conditions, but did not increase normally after BIC injections.

In summary, BIC and MUS caused small but significant changes in the resting ventilatory patterns of quiet bats: BIC shortened inspirations whereas MUS lengthened expirations (Fig. 7A,B). Under normal conditions, inspirations became uniformly shorter during bouts of calling than during quiet breathing $(7 A, C, E)$, but we observed no significant effects of either BIC or MUS on inspiration duration during calling, either with or without performing DSC (Fig. 7C,E). Finally, expiration durations were significantly lengthened by MUS while the bat emitted one call per breath (Fig. 7D) but not while the bat emitted two calls per breath (Fig. 7F). Oppositely, BIC caused a significant 
reduction in expiration duration during DSC performance (Fig. $7 F$ ) but had no significant effects on expiration duration while the bats emitted one call per breath (Fig. 7D).

\section{Discussion}

Somewhere within the mammalian midbrain, the descending vocal motor pathway is presumed to divide into separate laryngeal and respiratory components (Jürgens, 2002). Precisely how and where in the brain the vocal motor pathways interact with the respiratory rhythm centers is unknown. However, it is known that the lateral, and especially the ventrolateral region of the $\mathrm{PB}$ is centrally involved in the coordination of respiratory reflexes (Fung and St. John, 1994; Feldman et al., 2003; Potts et al., 2005), and that the $\mathrm{PB}$ is generally considered an integral component of the mammalian vocal motor pathway (Moga and Caper, 1990; Farley et al., 1992; Chamberlin and Saper, 1994; Sakamoto et al., 1997). Thus, just as appears to be the case in birdsong control (Wild et al., 1990), several lines of evidence suggest that the mammalian PB may be centrally involved in the coordination of vocal and respiratory motor patterns.

Here, we presented evidence that pharmacological manipulation of $\mathrm{GABA}_{\mathrm{A}}$-type synaptic activity in the lateral PB caused a very specific change in vocal motor patterns that could be attributed to an equally specific deficiency in the respiratory control circuitry. Specifically, after BIC injections into the lateral PB, both calls and intercall intervals became shorter and the bats no longer emitted two calls per breath in response to frequencyshifted playback (Fig. 5E). MUS had the opposite effect, increasing both call durations and intercall intervals. We believe that these changes can be accounted for by a change in the underlying mechanisms coordinating breathing patterns with calling. During DSC, the expiratory phase must be lengthened to accommodate the additional calls (Fig. 6) associated with this behavior (Smotherman and Metzner, 2005), and it follows that this is achieved by postponing the next inspiratory onset. After BIC injections, however, inspirations were no longer postponed sufficiently to allow for more than one syllable per breath. Alternatively, MUS extended expiratory durations, presumably by postponing inspiratory onset.

Notably, although BIC caused a slight increase in the quiet breathing rate (Fig. 6), the resulting breathing rates cannot be characterized as hyperpneic, nor did MUS induce apnea. This is consistent with previous conclusions that although the lateral PB has a potent influence on breathing, it does not appear to play a central role in respiratory rhythmogenesis. We conclude that reduced GABAergic input to neurons in the lateral PB, such as that caused by blockage by BIC, shortened mean call durations and sharply reduced the bat's ability to emit more than one call per breath. We hypothesize that this effect arose as a result of premature inspiratory on-switching caused by BIC in the lateral PB.

$\mathrm{BIC}$ and MUS injections into the lateral $\mathrm{PB}$ had in fact quite subtle, although significant, effects on temporal vocal motor patterns in horseshoe bats; mean call durations were reduced by only $16 \%$ (Fig. 4) and the call structure remained unchanged. It was only because horseshoe bats emit longer vocalizations than other bats, and because these bats could be provoked through controlled acoustic stimuli to emit longer, more complex vocal motor patterns during DSC that we were able to recognize that a change had occurred in the coordination between calling and breathing during these experiments. By using noninvasive breathing measurements in combination with relatively longlasting drugs (BIC and MUS), we were able to accurately charac- terize these subtle changes in the relationship between calling and breathing.

A central question is whether vocal motor patterns were, in fact, terminated by premature inspirations or were instead breath patterns altered indirectly in response to pharmacological changes in central vocal motor patterns. Two lines of evidence support the former conclusion: first, both BIC and MUS caused subtle changes in quiet breathing, and second, although MUS caused a significant increase in expiration durations surrounding the emissions of one call per breath, the increase was not matched by proportionally longer calls; calls were longer, but the increase in call durations was much less than the increase in expiration durations. The effects of both BIC and MUS on quiet breathing patterns are consistent with previous reports that the lateral $\mathrm{PB}$ in rodents sends excitatory projections to the medullary respiratory centers, and that stimulating these projections shortens both inspiratory and expiratory durations by promoting inspiratory onset and enhancing phrenic nerve discharge (Potts et al., 2005). We hypothesize that in our experiments the application of BIC increased neuronal activity in the lateral $\mathrm{PB}$, thereby enhancing inspiratory drive and mimicking the effects of excitatory stimulation in the lateral PB as described previously (Rybak et al., 2004a,b; Potts et al., 2005). A BIC-induced increase in phrenic nerve discharge would account for the shorter inspiratory durations observed during quiet breathing. Conversely, MUS presumably suppressed activity in these neurons, thereby extending expiratory durations by postponing inspiratory onset both during quiet breathing and during bouts of calling.

$\mathrm{PB}$ neurons are known to regulate respiratory phase switching in support of normal respiratory reflexes as well as during a phenomenon known as locomotor-respiratory coupling (Bramble and Carrier, 1983; Speakman and Racey, 1991; Funk et al., 1992). Electrical or chemical stimulation of the lateral parabrachial nucleus can cause premature initiation of inspiration and increased phrenic nerve discharge (Cohen, 1971; Miura and Takayama, 1991; Chamberlin and Saper, 1994), indicating that neurons in the lateral $\mathrm{PB}$ regulate inspiratory on-switching. Potts et al. (2005) demonstrated that in rats, respiratory entrainment to locomotor activity was achieved via excitatory somatosensory feedback to the lateral parabrachial nucleus near the ventrolateral border of the superior cerebellar peduncle within the PB, which in turn sends projections to the medullary respiratory rhythm centers; glutamate injections into this region of the PB caused premature inspiratory on-switching, whereas pharmacological inactivation blocked locomotor-respiratory entrainment. Specific functional domains of the parabrachial nucleus have not been delineated in bats to the same extent as in rodents; however, our injection coordinates overlapped with the lateral border of the cerebral cerebellar peduncle within the PB (Figs. 1, 2); thus, our results may reflect the activity of analogous groups of neurons. Previous reports have made it clear that glutamatergic synaptic activity in the lateral $\mathrm{PB}$ is involved in locomotorrespiratory coupling (Miura and Takayama, 1991; Chamberlin and Saper, 1994; Potts et al., 2005), and we presume that it may also contribute to vocal-respiratory coupling in bats. Experiments are underway that explore how excitatory and inhibitory inputs may be integrated in the lateral PB to facilitate vocalrespiratory coupling during echolocation by bats.

The significance for these results lies in the possibility that normal vocal-respiratory coupling in mammals requires intact somatosensory feedback loops similar if not identical to 
the ones that mediate other forms of locomotor-respiratory coupling.

From these experiments, we cannot say whether or not the respiratory phase-switching observed here was being modulated by either descending vocal motor commands to the PB or from somatosensory feedback arising from the larynx. Indeed both are likely to contribute under normal circumstances (supplemental Fig. 1, available at www.jneurosci.org as supplemental material). For example, the respiratory component of the vocal motor pathway must also include activation of the lateral abdominal muscles to generate the high subglottic pressures needed to vocalize (Jürgens and Schriever, 1991; Lancaster and Speakman, 2001), and these likely derive from central vocal motor commands. However, the evidence presented here demonstrates that vocalrespiratory coupling can be disrupted by pharmacological manipulations of a region of the $\mathrm{PB}$ that has also been shown to be centrally involved in other forms of locomotor-respiratory coordination that rely on somatosensory feedback control. Thus, one of the apparent roles of the PB during vocalizing may be to provide an indirect link between breathing and vocalizing via normal respiratory reflexes. If vocalizing and breathing are rhythmically coupled via highly conserved respiratory reflexes, then it can be safely assumed that the central mechanisms by which horseshoe bat vocalizations entrain respiratory rhythms are very likely to be the same ones used by other highly vocal mammals. Therefore, these results provide a clear indication of how and where in the brain basic respiratory reflexes may contribute to the normal flow of speech in humans.

\section{References}

Altarifi A, Badr MS, Tzelepis GE (2003) Maximal dynamic expiratory pressures with fast and slow inspirations. Eur J Appl Physiol 89:74-78.

Ashmore RC, Wild JM, Schmidt MF (2005) Brainstem and forebrain contributions to the generation of learned motor behaviors for song. J Neurosci 25:8543-8554.

Bramble DM, Carrier DR (1983) Running and breathing in mammals. Science 219:251-256.

Chamberlin NL, Saper CB (1994) Topographic organization of respiratory responses to glutamate microstimulation of the parabrachial nucleus in the rat. J Neurosci 14:6500-6510.

Cohen MI (1971) Switching the respiratory phases and evoked phrenic responses produced by rostral pontine electrical stimulation. J Physiol (Lond) 217:133-158.

Ezure K, Tanaka I, Miyazaki M (1998) Pontine projections of pulmonary slowly adapting receptor relay neurons in the cat. NeuroReport 9:411-414.

Farley GR, Barlow SM, Netsell R (1992) Factors influencing neural activity in parabrachial regions during cat vocalizations. Exp Brain Res 89:341-351.

Feldman JL (1986) Neurophysiology of breathing in mammals. In: Handbook of physiology. The nervous system, intrinsic regulatory systems of the brain. (Brookhart JM, Mountcastle VB, eds). Bethesda, MD: Am Physiol Soc.

Feldman JL, Gautier H (1976) Interactions of pulmonary afferents and pneumotaxic center in control of respiratory pattern in cats. J Neurophysiol 39:31-44.

Feldman JL, Mitchell GS, Nattie EE (2003) Breathing: rhythmicity, plasticity, chemosensitivity. Annu Rev Neurosci 26:239-266.

Fung ML, St. John WM (1994) Separation of multiple functions in the ventilatory control of pneumotaxic mechanisms. Respir Physiol 96:83-98.

Funk GD, Milsom WK, Steeves JD (1992) Coordination of wingbeat and respiration in the Canada goose. I. Passive wing flapping. J Appl Physiol 73:1014-1024.

Hartley DJ, Suthers RA (1988) The acoustics of the vocal tract in the horseshoe bat, Rhinolophus hildebrandti. J Acoust Soc Am 84:1201-1213.

Jürgens U (2002) Neural pathways underlying vocal control. Neurosci Biobehav Rev 26:235-258.
Jürgens U, Schriever S (1991) Respiratory muscle activity during vocalization in the squirrel monkey. Folia Primatol 56:121-132.

Kent RD (1997) Speech production: motor control, brain research, and fluency disorders (Hulstijn W, Peters HFM, van Lieshout PHHM, eds), pp 13-36. Amsterdam: Elsevier.

Kubke MF, Yazaki-Sugiyama Y, Mooney R, Wild JM (2004) Physiology of neuronal subtypes in the respiratory-vocal integration nucleus retroambigualis of the male zebra finch. J Neurophysiol 94:2379-2390.

Lancaster WC, Speakman JR (2001) Variations in respiratory muscle activity during echolocation when stationary in three species of bat (Microchiroptera: Vespertilionidae). J Exp Biol 204:4185-4197.

Metzner W (1993) An audio-vocal interface in echolocating horseshoe bats. J Neurosci 13:1899-1915.

Metzner W (1996) Anatomical basis for audio-vocal integration in echolocating horseshoe bats. J Comp Neurol 368:252-269.

Metzner W, Zhang SY, Smotherman MS (2002) Doppler-shift compensation behavior in horseshoe bats revisited: auditory feedback controls both a decrease and an increase in call frequency. J Exp Biol 205:1607-1616.

Miura A, Takayama K (1991) Circulatory and respiratory responses to glutamate stimulation of the lateral parabrachial nucleus of the cat. J Auton Nerv Syst 21:195-202.

Moga HH, Caper CB (1990) Connections of the parabrachial nucleus with the nucleus of the solitary tract and the medullary reticular formation in the rat. J Comp Neurol 293:540-580.

Nakazawa K, Shiba K, Satoh I, Yoshida K, Nakajima Y, Konno A (1997) Role of pulmonary afferent inputs in the vocal on-switch in the cat. Neurosci Res 29:49-54.

Neuweiler G, Metzner W, Heilmann U, Rubsamen R, Eckrich M, Costa HH (1987) Foraging behaviour and echolocation in the rufous horseshoe bats, Rhinolophus rouxi, of Sri Lanka. Behav Ecol Sociobiol 20:53-67.

Palecek F (1969) Measurements of ventilatory mechanics in the rat. J Appl Physiol 27:149-156.

Paxinos G, Watson C (1998) The rat brain in stereotaxic coordinates, Ed 4. San Diego: Academic.

Potts JT, Paton JFR (2001) Phase-coupling of the respiratory network by somatosensory receptors during locomotion. Respir Res 2:7.

Potts JT, Rybak IA, Paton JFR (2005) Respiratory rhythm entrainment by somatic afferent stimulation. J Neurosci 25:1965-1978.

Rübsamen R, Betz M (1986) Control of echolocation pulses by neurons of the nucleus ambiguus in the rufous horseshoe bat, Rhinolophus rouxi. I. Single unit recordings in the ventral motor nucleus of the laryngeal nerves in spontaneously vocalizing bats. J Comp Physiol A Neuroethol Sens Neural Behav Physiol 159:675-687.

Rybak IA, Shevtsova NA, Paton JF, Pierrefiche O, St. John WM, Haji A (2004a) Modeling respiratory rhythmogenesis: focus on phase-switching mechanisms. Adv Exp Med Biol 551:189-194.

Rybak IA, Shevtsova NA, Paton JFR, Dick TE, St. John WM, Mörschelm M, Dutscmann M (2004b) Modeling the ponto-medullary respiratory network. Respir Physiol Neurobiol 143:307-319.

Sakamoto T, Nonaka S, Katada A (1997) Control of respiratory muscles during speech and vocalization. In: Neural control of the respiratory muscles. (Miller AD, Bianchi AL, Bishop BP, eds), pp 249-258. New York: CRC.

Schnitzler HU (1968) Die ultraschallortungslaute der hufeisennasenfledermäuse (Chiroptera, Rhinolophidae) in verschiedenen orientierungssituationen. Z Vergl Physiol 57:376-408.

Schnitzler HU (1973) Control of Doppler shift compensation in the Greater Horseshoe Bat, Rhinolophus ferrumequinum. J Comp Physiol 82:79-92.

Schuller G (1986) Influence of echolocation pulse rate on Doppler-shift compensation control system in the Greater Horseshoe Bat. J Comp Physiol A Neuroethol Sens Neural Behav Physiol 158:239-246.

Schuller G, Radtke-Schuller S (1990) Neural control of vocalization in bats: mapping of brainstem areas with electrical microstimulation eliciting species-specific echolocation calls in the rufous horseshoe bat. Exp Brain Res 79:192-206.

Schuller G, Rübsamen R (1981) Laryngeal nerve activity during pulse emission in the CF-FM bat, Rhinolophus ferrumequinum. I. Superior laryngeal nerve. J Comp Physiol 143:317-321.

Schuller G, Beuter K, Schnitzler HU (1974) Response to frequency-shifted artificial echoes in the bat, Rhinolophus ferrumequinum. J Comp Physiol 89:275-286.

Schuller G, Beuter K, Rübsamen R (1975) Dynamic properties of the com- 
pensation system for Doppler-shifts in the bat, Rhinolophus ferrumequinum. J Comp Physiol 97:113-125.

Schuller G, Radtke-Schuller S, Betz M (1986) A stereotaxic method for small animals using experimentally determined reference profiles. J Neurosci Methods 18:339-350.

Smotherman M, Metzner W (2005) Auditory feedback control of temporal call patterns in echolocating horseshoe bats. J Neurophysiol 93:1295-1303.

Smotherman M, Zhang S, Metzner W (2003) A neural basis for auditory feedback control of vocal pitch. J Neurosci 23:1464-1477.

Speakman JR, Racey PA (1991) No cost of echolocation for bats in flight. Nature 350:421-423.

Sturdy CB, Wild JM, Mooney R (2003) Respiratory and telencephalic modulation of vocal motor neurons in the zebra finch. J Neurosci 23:1072-1086.

Suthers RA (1988) The production of echolocation signals by birds and bats.
In: Animal sonar: processes and performance (Nachtigall PE, Moore PWB, eds), pp 23-45. New York: Plenum.

Suthers RA, Hartley DJ, Wenstrup JJ (1988) The acoustic role of tracheal chambers and nasal cavities in the production of sonar pulses by the horseshoe bat, Rhinolophus hildebrandti. J Comp Physiol A Neuroethol Sens Neural Behav Physiol 162:799-813.

Suthers RA, Goller F, Wild JM (2002) Somatosensory feedback modulates the respiratory motor program of crystallized birdsong. Proc Natl Acad Sci USA 99:5680-5685.

Swanson LW (1992) Brain maps: structure of the rat brain. Amsterdam; New York: Elsevier.

Tian B, Schnitzler HU (1997) Echolocation signals of the greater horseshoe bat (Rhinolophus ferrumequinum) in transfer flight and during landing. J Acoust Soc Am 101:2347-2364.

Wild JM, Arends JJ, Zeigler HP (1990) Projections of the parabrachial nucleus in the pigeon (Columba livia). J Comp Neurol 293:499-523. 